\title{
Global Energy Optimization Strategy Based on Delay Constraints in Edge Computing Environment
}

\author{
Xianzhong Tian \\ Computer Science and Technology \\ Zhejiang University of Technology \\ Hangzhou, China \\ txz@zjut.edu.cn
}

\author{
Lu Zhou \\ Computer Science and Technology \\ Zhejiang University of Technology \\ Hangzhou, China \\ 18870276896@163.com
}

\author{
Ting Xu \\ Computer Science and Technology \\ Zhejiang University of Technology \\ Hangzhou, China \\ 2111812051@zjut.edu.cn
}

\begin{abstract}
Edge Computing is one of the core technology of 5G networks. Edge computing deploys servers at the edge of the wireless access network, sinking cloud computing capabilities to the edge of the network, sharing the computing pressure of mobile users nearby, and improving the computing power of the entire network. Energy consumption is one of the important research issues of edge computing. At present, research on edge computing focuses on the energy consumption of terminal device, while little attention is paid to the energy consumption of edge servers. In this paper, considering the above two kinds of energy consumption, a global energy optimization strategy based on delay constraint in edge computing environment is proposed. Specifically, first, we use queuing theory to analyze the average delay of each terminal device and edge cloud processing computing tasks in the Internet of Things network, and the average delay of the entire system processing computing tasks. Secondly, we use the average delay as a constraint to establish a mathematical model for minimizing the total energy consumption of the device and the server. Then, we design a genetic algorithmbased offloading computation optimization algorithm to solve the above problems, so as to obtain the number of running servers in the edge cloud and the offload probability of IoT devices. Finally, the goal of minimizing the energy consumption of the overall system under the time delay constraint is achieved. The simulation experiment verifies the effectiveness of the energy optimization strategy.
\end{abstract}

\section{CCS CONCEPTS}

- Networks $\rightarrow$ Cloud Computing;

\section{KEYWORDS}

SDN; IoT; edge computing;computing offloading

\section{ACM Reference Format:}

Xianzhong Tian, Lu Zhou, and Ting Xu. 2021. Global Energy Optimization Strategy Based on Delay Constraints in Edge Computing Environment. In Proceedings of the 24th ACM International Conference on Modeling, Analysis and Simulation of Wireless and Mobile Systems (MSWiM '21), November

Permission to make digital or hard copies of all or part of this work for personal or classroom use is granted without fee provided that copies are not made or distributed for profit or commercial advantage and that copies bear this notice and the full citation on the first page. Copyrights for components of this work owned by others than ACM must be honored. Abstracting with credit is permitted. To copy otherwise, or republish, to post on servers or to redistribute to lists, requires prior specific permission and/or a fee. Request permissions from permissions@acm.org.

MSWiM '21, November 22-26, 2021, Alicante, Spain

(C) 2021 Association for Computing Machinery.

ACM ISBN 978-1-4503-9077-4/21/11 \$\$15.00

https://doi.org/10.1145/3479239.3485692
22-26, 2021, Alicante, Spain. ACM, New York, NY, USA, 8 pages. https: //doi.org/10.1145/3479239.3485692

\section{INTRODUCTION}

Edge computing is one of the core technologies of $5 \mathrm{G}$ networks and has received extensive attention in recent years. Edge Computing deploys servers at the edge of the wireless access network(such as small base stations, macro base stations, cellular base stations, and WiFi access points), sinking cloud computing capabilities to the edge of the network, sharing the computing pressure of mobile users nearby, and improving the computing capability of the entire network[1]. Compared with traditional cloud computing, although the edge cloud does not have the powerful computing capability of the central cloud, it is closer to the terminal device and does not need to be transmitted through the backbone network, which greatly shortens the transmission distance, thereby reducing energy consumption[9].

Energy consumption is one of the key issues of edge computing. At present, the research on the energy consumption of edge computing is mainly concentrated on the terminal device side. Part of it is to minimize the total energy consumption of the device by constraining the task computation time. Literature [17] proposed a low-complexity energy discretization algorithm, which can save nearly $82.7 \%$ of the energy of mobile devices. The other part designs the calculation delay and the energy consumption of the device into one quantity, and then minimizes this quantity. Literature [18] divides the complex task into several subtasks, and implements an effective offloading strategy to reduce the execution time of the application program and the weighted sum of the energy consumption of the device. Few studies have focused on the energy consumption on the edge server side. Although the edge server can be directly connected to a wired power supply and does not need to be powered by a battery like a wireless terminal, its calculation amount is much higher than terminal device, so its energy consumption is not negligible.

The main reason that affects the energy consumption of edge servers is the number of edge servers turned on. Generally, the power of a server at no load exceeds $50 \%$ of the peak power, and the average server utilization is usually only $10 \%-50 \%$. The energy consumption of a server running at $20 \%$ performance may be equivalent to $80 \%$ of its energy consumption of full load[2]. In other words, the energy consumption of a server is not directly proportional to its load. The energy consumption is very large when it is empty and cannot be ignored. The solution is to shut down the redundant edge servers. Specifically, according to the workload of the server, activate the necessary number of servers and shut down the 
redundant servers. However, the load of the edge server is directly related to the computing offloading decision of the terminal device. The more offloads, the heavier the load on the edge server. On the one hand, it is hoped to offload as many edge servers as possible to reduce the computing delay, but on the other hand, this increases the computing load of the edge servers and activates more servers. How to weigh the relationship between the two to optimize the overall performance of the system is a problem worthy of study.

This paper jointly considers the energy consumption of the IoT device side and the edge server side, and designs a global energy optimization computing offloading strategy based on delay constraints. Through reasonable arrangements to calculate the probability of offloading and the number of server activated, the global energy consumption of the system is minimized. The main contributions of this paper are summarized as follows:

- Analyze the average calculation delay of the IoT device side and the edge server side through the queuing theory, and model the overall calculation delay of the computing system.

- Use the above-mentioned overall calculation delay to model the global energy consumption minimization problem under the delay constraints.

- Use the genetic algorithm to solve the above-mentioned global energy consumption minimization problem, and obtain the computing offloading strategy of the IoT device and the activation number of edge servers.

The rest of this article is organized as follows. The second part reviews related work. The third part introduces the system model. The fourth part formulates optimization problems. The fifth part solves the optimization problem. The sixth part shows the experimental results. The seventh part is the conclusion.

\section{RELATED WORK}

At Present, the existing literature on edge computing research can be roughly divided into three categories: 1 ) Research with the goal of minimizing computing delay. Considering that the edge cloud may be overloaded, it will increase the delay of computing tasks. Literature [11] and [7] both proposed that the central cloud and the edge cloud collaborate to share computing tasks to reduce the task delay. The literature [7] proposed an edge cloud framework based on software-defined network, and adopted Q-Learning and cooperative Q-Learning algorithm to solve the problem of offloading decision. In [12], in D2D-based mobile edge computing scenarios, whether it is edge cloud or terminal device, task can be offloaded to it. Literature [15] uses queuing theory to analyze the average response time of tasks in the system, and proposes a greedy algorithm, which saves $20 \%-30 \%$ of the time. 2) Research aimed at minimizing energy consumption. Literature [6], [4], [10] all use deep reinforcement learning algorithms to solve the problems of computing offloading and resource allocation, which greatly reduces the energy consumption of terminal devices. In [6], in order to further reduce the complexity of the algorithm, an adaptive process is proposed, which can dynamically adjust the parameters of the algorithm. Literature [4] established the application model as a directed acyclic graph, and then determined the offloading decision and resource allocation plan of each subtask. Literature [10] proposed a mobile edge computing system with energy capture.
Literature [14] proposed OTCA and OECAA algorithms for two subproblems. Simulation experiments show that the OTCA algorithm reduces energy consumption by $53.14 \%$ compared to the random algorithm, and the OECAA algorithm has higher performance than the OTCA algorithm. 3) Research aimed at optimizing time delay and energy consumption at the same time. Literature [16], [5], [8] solve the problem of computing offload and resource allocation in edge computing systems. Literature [16] proposed an integrated framework and energy- aware offloading scheme to minimize the weighted sum of energy consumption and delay by optimizing offloading decision-making, power allocation, channel allocation, and computing resource allocation. Literature [5] proposed an improved NSGA- algorithm to solve the problem of unloading decision-making for IoT devices. Literature [8] is based on Lyapunov optimization theory, which converts the delay and energy consumption trade-off problem into a series of deterministic problems in each time block.

Although the above-mentioned research involves the energy consumption of the edge system, most of the literature focuses on the energy consumption of the devices, and little attention is paid to the energy consumption of the edge server. With the increase of IoT devices, more and more computing tasks are offloaded to the edge cloud, which increases the energy consumption of edge servers. In the mobile edge computing system, terminal equipment and edge cloud are both indispensable components. We cannot only consider one side and ignore the other. This paper jointly considers the overall energy consumption of edge cloud and IoT devices, and studies global energy optimization strategy based on delay constraints.

\section{SYSTEM MODEL}

\subsection{Scenario Description}

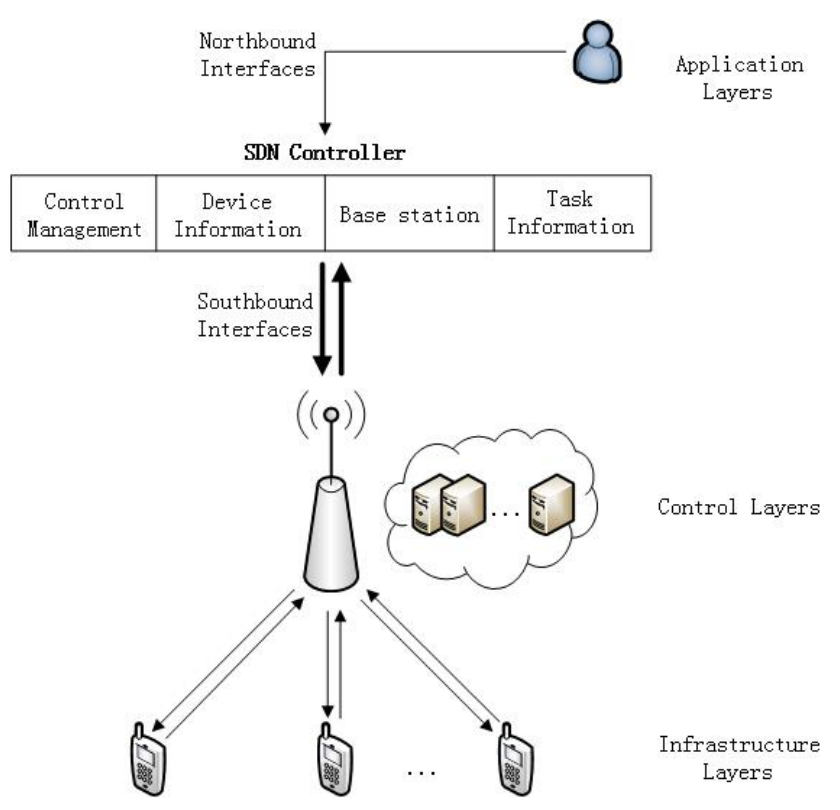

Figure 1: An SDN-based IoT network architecture. 
As shown in Figure 1, this paper studies an IoT network architecture composed of $m$ devices and a small cell base station integrated with edge cloud. Among them, the edge cloud is equipped with multiple physical servers to provide computing services for energyconstrained IoT devices. Each IoT device can choose to offload part of the computing tasks to the edge cloud for computing, and the remaining tasks for local computing. This IoT network architecture is divided into three layers, namely the application layer, the control layer, and the infrastructure layer. The control layer is composed of a centralized SDN controller. The SDN controller interacts with the application layer and the infrastructure layer through the southbound interface and the northbound interface.

Each IoT device executes an application, which will generate a series of computing tasks. Assuming that the arrival rate of computing tasks of devices $i \in\{1,2, \ldots, m\}$ obeys Poisson distribution with rate $\lambda_{i}$, and the types of computing tasks $A_{i}=\left\{b_{i}, c_{i}, T_{i}\right\}$ generated by the same device are same. Where $b_{i}$ is the size of computing task, $c_{i}$ is the CPU cycles needed for execution, and $T_{i}$ is the maximum tolerance delay. Therefore, the task request data packet of the device usually includes $\lambda_{i}, b_{i}, c_{i}, T_{i}$.

The entire network architecture is under the SDN controller, and the SDN controller interacts with the base station and the device by sending Open-Flow messages to the base station and the Internet of Things devices, so as to globally control the edge of the entire network[13]. When a task of the IoT device arrives, it sends a task request to the nearest base station, and then the base station sends the request to the SDN controller. After the SDN controller receives the task request information, it will update all the information tables. Finally, the SDN controller makes task offloading strategies for IoT devices and resource allocation strategies for edge clouds based on task delay constraints and energy consumption. Since the signaling data packet is very small, we can ignore the transmission energy consumption of this process[3].

\subsection{Joint Queuing Network Model}

As shown in Figure 2, the offloading model is divided into two parts, one is the communication module, and the other is calculation module. After the IoT device receives the computing offloading decision signaling, part of the computing tasks will enter the communication module queue of the device $i$ at a rate $p_{i} \lambda_{i}$, where the offload probability is $p_{i}$. Then they will be transmitted to the edge cloud for calculation through the communication module. The rest will go to the local calculation.

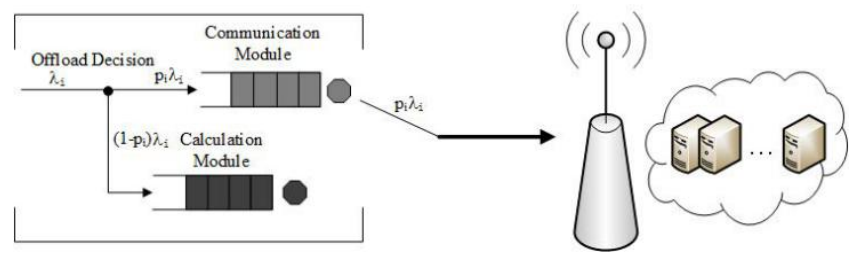

Figure 2: Offloading model.

We apply queuing theory to model the average response time of computing tasks. In our architecture, the processing queue of the communication module of each device can be regarded as the $M / M / 1$ queue model. The edge cloud has more than one server. It is a small cell base station system composed of multiple servers, so the service process of the edge cloud can be regarded as an $\mathrm{M} / \mathrm{M} / \mathrm{K}$ queue model. Suppose there are IoT devices in the system that need to share the edge cloud. We define the set of the arrival rate of the computing tasks of each IoT device in the system as $\lambda=\left\{\lambda_{1}, \lambda_{2}, \ldots, \lambda_{m}\right\}$, the set of offloading probability $p=\left\{p_{1}, p_{2}, \ldots, p_{m}\right\}$, and the set of the service rate of the communication module as $\mu=\left\{\mu_{1}, \mu_{2}, \ldots, \mu_{m}\right\}$, A computing task that is offloaded to the edge cloud for computing needs to undergo two service processes: one is the transmission service provided by the communication module in the device, and the other is the computing service provided by the edge cloud.We call them service 1 and service 2 .

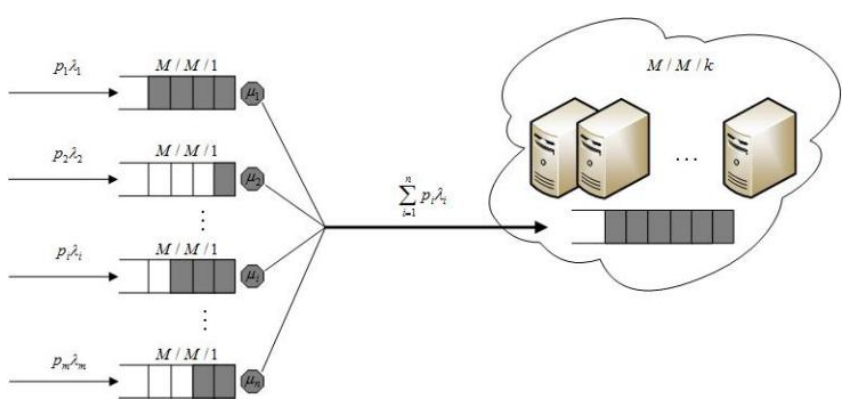

Figure 3: Joint queuing network model.

We model the multi-device offloading system as a joint network queuing model,as shown in Figure 3.Denote $P_{\left\{x_{i}\right\}}, i \in\{1,2, \ldots, m+1\}$ as the steady state probability of the model,where $x_{i}$ is the number of the tasks in one of the queues.The queue length of the model is:

$$
\left.L=\sum_{x_{i}}\left(x_{1}+x_{2}+\cdots+x_{m+1}\right) P_{\{} x_{i}\right\}
$$

The transmission rate between the device $i$ and the base station is $r_{i}=B \log _{2}\left(1+\frac{\rho_{i}^{t} h_{i}}{\delta}\right)$, where $B$ represents the channel bandwidth, $\rho_{i}^{t}$ represents the transmission power of the device, $h_{i}$ represents the channel gain between the device and the BS and $\delta$ is the Gaussian noise.

It can be easily proved that the departure process of computing tasks at the communication module is also a Poisson process with a rate of $p_{i} \lambda_{i}$, where $p_{i} \lambda_{i} \leq \mu_{i}$. And it can be inferred that the number of tasks in service 1 is independent of the number of tasks that left from service 1 in the past, and these tasks that left in the past from service 1 constitute the arrival process of service 2 . Therefore, the number of tasks in service 1 and service 2 is independent.

Thus, the arrival rate of the edge cloud is $\sum_{i=1}^{m} p_{i} \lambda_{i}$. And each module is independent of each other, so:

$$
P_{\left\{x_{i}\right\}}=\Pi_{i=1}^{m+1} P_{x_{i}}
$$

where, $P_{x_{i}}$ is the steady state probability of one of the queues. Then, we can rewrite formula (1):

$$
L=\sum_{m+1} \sum_{x_{i}} x_{i} P_{x_{i}}
$$

A close observation of (3) shows that $L_{i}=\sum_{x_{i}} x_{i} P_{x_{i}}$ is the queue length of one of the queues. 
Theorem 1. Under the condition of $p_{i} \lambda_{i} \leq \mu_{i}$, the queue length of communication module $i$ at steady state

$$
L_{i}=\frac{p_{i} \lambda_{i}}{\mu_{i}-p_{i} \lambda_{i}}
$$

Proof.

1 Write the equilibrium equation:

Status Departure rate $=$ Arrival rate

$0 \quad p_{i} \lambda_{i} P_{0}=\mu_{i} P_{1}$

$x_{i}, x_{i}>0\left(p_{i} \lambda_{i}+\mu_{i}\right) P_{n}=p_{i} \lambda_{i} P_{n-1}+\mu_{i} P_{n+1}$

2 Rewrite the equation set:

$$
\begin{aligned}
& P_{1}=\frac{p_{i} \lambda_{i}}{\mu_{i}} P_{0} \\
& P_{x_{i}+1}=\frac{p_{i} \lambda_{i}}{\mu_{i}} P_{x_{i}}+\left(P_{x_{i}}-\frac{p_{i} \lambda_{i}}{\mu_{i}} P_{x_{i}-1}\right)
\end{aligned}
$$

3 Solve according to $P_{0}$

$$
\begin{aligned}
& P_{0}=P_{0} \\
& P_{1}=\frac{p_{i} \lambda_{i}}{\mu_{i}} P_{0} \\
& P_{2}=\frac{p_{i} \lambda_{i}}{\mu_{i}} P_{1}+\left(P_{1}-\frac{p_{i} \lambda_{i}}{\mu_{i}} P_{0}\right)=\frac{p_{i} \lambda_{i}}{\mu_{i}} P_{1}=\left(\frac{p_{i} \lambda_{i}}{\mu_{i}}\right)^{2} P_{0} \\
& P_{3}=\frac{p_{i} \lambda_{i}}{\mu_{i}} P_{2}+\left(P_{2}-\frac{p_{i} \lambda_{i}}{\mu_{i}} P_{1}\right)=\frac{p_{i} \lambda_{i}}{\mu_{i}} P_{2}=\left(\frac{p_{i} \lambda_{i}}{\mu_{i}}\right)^{2} P_{0} \\
& P_{x_{i}+1}=\frac{p_{i} \lambda_{i}}{\mu_{i}} P_{x_{1}}+\left(P_{x_{i}}-\frac{p_{i} \lambda_{i}}{\mu_{i}} P_{x_{i}+1}\right)=\frac{p_{i} \lambda_{i}}{\mu_{i}} P_{x_{i}}=\left(\frac{p_{i} \lambda_{i}}{\mu_{i}}\right)^{x_{i}+1} P_{0}
\end{aligned}
$$

4 The sum of steady state probabilities is 1 , then

$$
\begin{aligned}
& 1=\sum_{x_{i}=0}^{\infty} P_{n}=\left(\frac{p_{i} \lambda_{i}}{\mu_{i}}\right)^{x_{i}+1} P_{0}=\frac{P_{0}}{1-\frac{p_{i} \lambda_{i}}{\mu_{i}}} \\
& P_{0}=1-\frac{p_{i} \lambda_{i}}{\mu_{i}} \\
& P_{x_{i}+1}=\left(\frac{p_{i} \lambda_{i}}{\mu_{i}}\right)^{x_{i}}\left(1-\frac{p_{i} \lambda_{i}}{\mu_{i}}\right)
\end{aligned}
$$

so the queue length of communication module $i$

$L_{i}=\sum_{x_{i}} x_{i} P_{x i}=\sum_{x_{i}}^{\infty} x_{i}\left(\frac{p_{i} \lambda_{i}}{\mu_{i}}\right)^{x_{i}}\left(1-\frac{p_{i} \lambda_{i}}{\mu_{i}}\right)=\frac{p_{i} \lambda_{i}}{\mu_{i}-p_{i} \lambda_{i}}$

Theorem 2. Assuming that the number of servers staying awake at the edge could is $k$, the service rate of each server is $\mu$. Then we can get the queue length of the edge cloud is

$$
L_{e}=\frac{\rho^{k} \rho^{\prime} P_{0}}{k !\left(1-\rho^{\prime}\right)^{2}}+\rho
$$

where, $\rho=\frac{\sum_{i=1}^{m} p_{i} \lambda_{i}}{\mu}, \rho^{\prime}=\frac{\sum_{i=1}^{m} p_{i} \lambda_{i}}{k \mu}, P_{0}=\frac{1}{\sum_{i=0}^{k-1} \frac{\rho^{i}}{i !}+\frac{\rho^{k}}{k !\left(1-\rho^{\prime}\right)}}$

Proof. Similar to the proof of Theorem 2, so no detailed proof will be given.

Therefore, according to formula (4) and formula (5), we can get the queue length of the joint queuing network:

$$
L=\sum_{m} L_{i}+L_{e}
$$

The average response time of the computing tasks in this joint queuing network can be obtained by the Little's Law:

$$
W=\frac{L}{\sum_{i=1}^{m} p_{i} \lambda_{i}}
$$

\subsection{Local Computing Model}

The service process of the local computing module also obeys the $\mathrm{M} / \mathrm{M} / 1$ queuing model[3]. As shown in figure 2, the computing tasks will enter the queue of the computing module of device $i$ at a rate of $\left(1-p_{i}\right) \lambda_{i}$, to accept local computing services. The service rate is $\mu_{i}^{l}$. According to the analysis of $\mathrm{M} / \mathrm{M} / 1$ in $B$, it can be deduced that the queue length of the computing module of device $i$ is

$L_{i}^{l}=\sum_{x_{i}} x_{i} P_{x_{i}}=\sum_{x_{i}}^{\infty}\left(\frac{\left(1-p_{i}\right) \lambda_{i}}{\mu_{i}^{l}}\right)^{x_{i}}\left(-\frac{\left(1-p_{i}\right) \lambda_{i}}{\mu_{i}}\right)=\frac{\left(1-p_{i}\right) \lambda_{i}}{\mu_{i}^{l}-\left(1-p_{i}\right) \lambda_{i}}$

The average response time of the computing task in the local calculation module of device $i$ can be obtained by the Little's Law:

$$
W_{l}=\frac{L_{i}^{l}}{\left(1-p_{i}\right) \lambda_{i}}=\frac{1}{\mu_{i}^{l}-\left(1-p_{i}\right) \lambda_{i}}
$$

\section{PROBLEM MODELING}

This article jointly considers the overall energy consumption of the IoT device and edge cloud. The ultimate goal is to minimize the total energy consumption of the system while meeting the delay constraints. The energy consumption on the IoT device side includes the energy consumption $E_{i}$ generated by the local computing module and the energy consumption $E_{t}$ generated by the data transmission of the communication

$$
\begin{gathered}
E_{i}=\rho_{i}^{l}\left(1-p_{i}\right) \lambda_{i} c_{i} \\
E_{t}=\rho_{i}^{t} \frac{p_{i} \lambda_{i} b_{i}}{\mu_{i}}
\end{gathered}
$$

where, $\rho_{i}^{l}$ is the energy consumption per cycle for local computing module of device $i$, and $\rho_{i}^{t}$ represents the transmission power.

The energy consumption in the edge cloud also includes two parts, namely the basic energy consumption of the server (even if there is no load, the wake-up server will generate energy consumption) and the energy consumption of performing computing tasks.

$$
E_{e}=\rho_{e}^{c} \sum_{i=1}^{m} p_{i} \lambda_{i} c_{i}+\rho_{e}^{s} k
$$

where, $\rho_{e}^{c}$ is the energy consumption per cycle for edge cloud performing computing tasks. $\rho_{e}^{s}$ represents the basic energy consumption of each server.

Because the edge server is wired and powered, the importance of its energy consumption is weaker than the importance of the energy consumption of wireless devices, so we design two parameters $\alpha$ and $\beta$ to describe their respective importance. Considering the energy consumption generated by IoT devices and the energy consumption generated by edge servers together, we formulate the problem as:

$$
\begin{gathered}
W_{l} \leq T_{i} \\
W \leq T_{i} \\
p_{i} \lambda_{i} \leq \mu_{i} \\
\sum_{i=1}^{m} p_{i} \lambda_{i} \leq k \mu \\
1 \leq k \leq s
\end{gathered}
$$$$
\mathbf{P}: \min _{p_{i}, k} \alpha \sum_{i=1}^{m}\left[E_{i}+E_{t}\right]+\beta E_{e}
$$ 
where, $E_{i}, E_{t}, E_{e}$ is obtained by formulas (10) to (12), $\alpha \sum_{i=1}^{m}\left[E_{i}+E_{t}\right]$ represents the energy consumption of the device side and $\beta E_{e}$ represents the energy consumption of the edge server side.

Constraint (14) is to ensure that the average response time of computing tasks in the local computing module of the device does not exceed the maximum tolerable delay. Constraint (15) is to ensure that the average response time of computing tasks in the joint queuing network does not exceed the maximum tolerable delay. Constraint (16) is to ensure that the arrival rate of computing tasks does not exceed the service rate of the communication module of the device. Constraint (17) is to ensure that the arrival rate of computing tasks does not exceed the service rate of the edge cloud, $s$ represents the number of edge servers. Constraints (18) indicates that the number of servers running in the edge cloud does not exceed the number of deployed servers.

\section{PROBLEM SOLVING}

Obviously, the problem proposed in (13) is NP-hard. To this end, we propose an optimization algorithm of computing offloading based on genetic algorithm (OACO: Optimization algorithm of computing offloading based on genetic algorithm) to solve this problem. First, we encode all the variables in the chromosome. Then select, cross and mutate the individual chromosome, and after multiple iterations, we can find the variable with the smallest target value. Combining the characteristics of genetic algorithm, the core of the algorithm can be summarized into the following:

\subsection{Chromosome Coding}

We binary code the offloading probability $p$ and the number of running servers $k$ in the problem. In genetic algorithms, each variable is a gene fragment on the chromosome. As shown in Figure 4, gene 0 represents the number of servers opened, that is, the variable $k$. Gene $i(i=1,2, \ldots, m)$ represent the offloading probability of each IoT devices, that is, the variable $p_{i},(i=1,2, \ldots, m)$. Assuming that in any chromosome, the length of fragment 0 is length 1 , and the length of fragment $i(i=1,2, \ldots, m)$ is length 2 . Therefore, the total length of the chromosome is $($ length $1+$ length $2 \cdot m)$.

For the binary code of the variable $k$, directly convert it into the form of a binary variable. Assuming that the length of length 1 is 5 and the value of variable $k$ is 3 , the binary code is 00011 . For the variable $p_{i}, p_{i} \in[0,1]$, first determine the precision of $p_{i}$, and then encode the variable as length 2 . Given a code of $p_{i}$, the corresponding offloading probability can be obtained through the formula $x^{t} \cdot \frac{1}{2^{\text {length }}-1}$. Among them, $x^{t}$ is the value converted from binary to decimal.

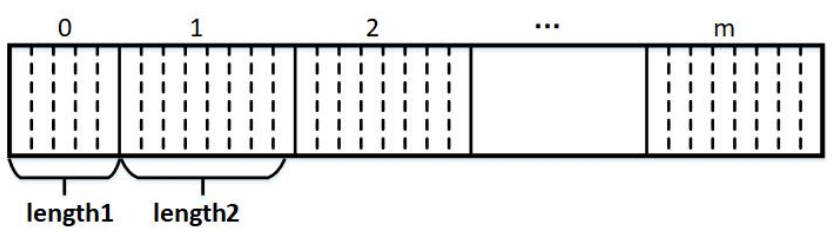

Figure 4: Chromosome structure.

\subsection{Fitness Function}

According to the objective problem $\mathbf{P}$, we define the objective function (19):

$$
f(p, k)=\alpha \sum_{i=1}^{m}\left[E_{i}+E_{t}\right]+\beta E_{e}
$$

Because the problem $\mathbf{P}$ is a minimization problem, the reciprocal of the objective function is defined as the fitness function (20). A set of candidate solutions is a chromosome. The smaller the objective function value, the higher the fitness value of the chromosome.

$$
\operatorname{Fit}(p, k)=\frac{1}{f(p, k)}
$$

\subsection{Crossover and Mutation}

The crossover of chromosomes only occurs on alleles. For example, fragment 0 can only exchange subsequences with fragment 0 . Mutation refers to the flipping of a binary bit in the chromosome, that is, 0 becomes 1 and 1 becomes $0 . p c$ and $p m$ are crossover probability and mutation probability. Randomly generate a random number between 0 and 1 . If the number is less than $p c$, chromosome crossover will occur. The same goes for chromosomal mutation.

\subsection{Select Operation}

The new chromosomes generated by crossover and mutation may not meet delay constraint. Therefore, when selecting the nextgeneration population, in addition to considering the value of the fitness function, we must also consider the delay constraint. Specifically, first, formula (7) is used to determine the delay constraint for newly generated individuals, and the chromosomes that do not meet the delay constraint are removed. Then, formula (20) is used to calculate the fitness function value of the chromosome that meets the requirements. After, according to the roulette algorithm, individuals with high fitness values are retained in the next-generation population.

The complete algorithm is shown in Algorithm 1. First, Initialize crossover probability, mutation probability, and randomly generate a population pop. At this time, a matrix of $s c \cdot($ length $1+$ length $2 \cdot m)$ is generated, where $s c$ represents the size of the population, that is, the number of chromosomes.

For each iteration, before calculating fitness, determine whether the individual meets the constraints. If the constraint conditions are met, the corresponding fitness value is calculated, otherwise, it will be recorded as 0 .

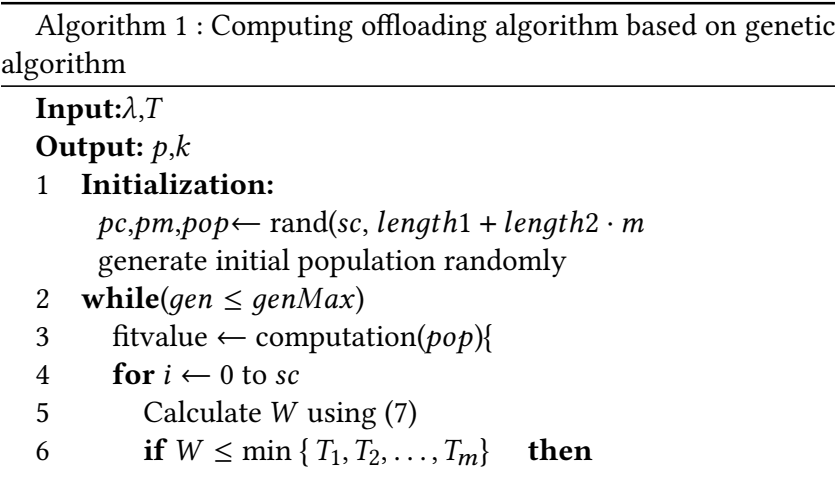




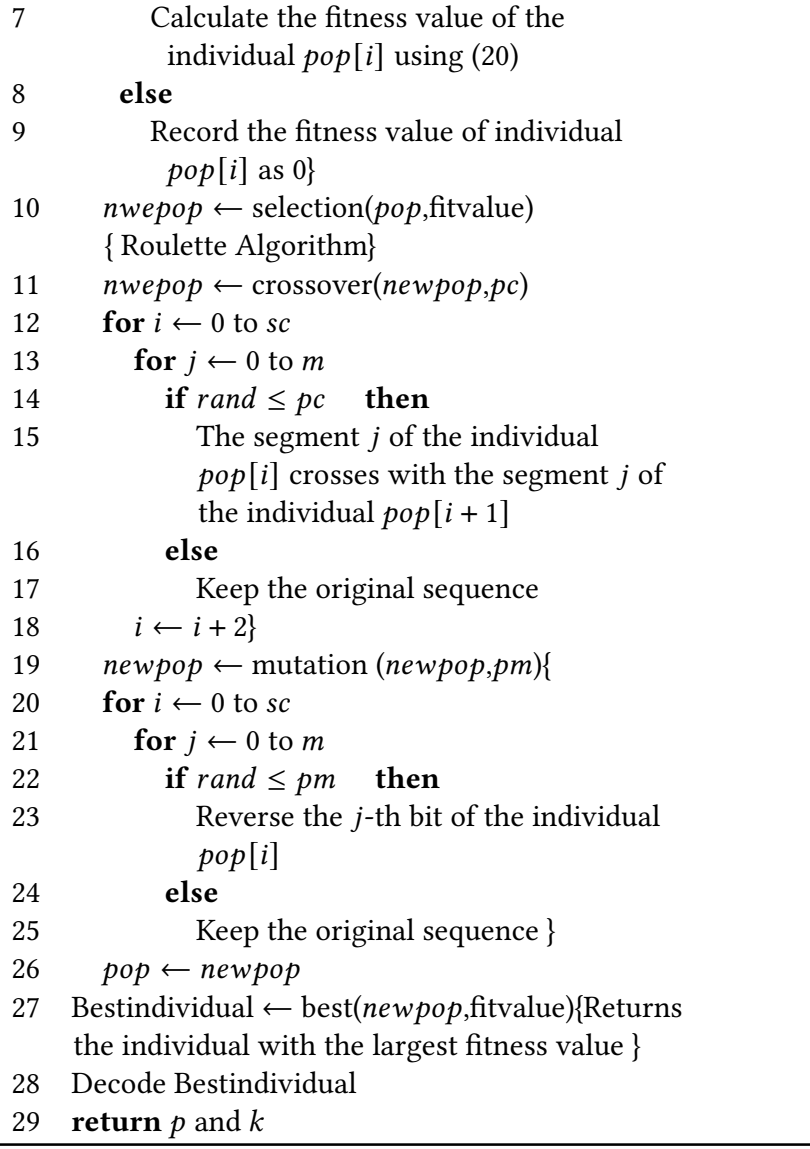

\section{PERFORMANCE ANALYSIS}

In this section, we use MATLAB R2020a to conduct simulation experiments to verify the effectiveness of the proposed algorithm for such problems.

\subsection{Experiment}

The experimental plan is set as follows:

- The entire Internet of Things network architecture consists of 1 edge cloud, 12 MEC servers and 100 IoT devices.

- Each IoT device randomly generates tasks, the data size of each task is $20-45 \mathrm{~KB}$, the number of CPU cycles required is $3^{-8}-5^{-8}$ cycle, and the maximum tolerable delay of the task is $1-3 \mathrm{~s}$.

- According to the current network status, each MEC server switches between on and off. When the MEC server is turned on, its basic energy consumption is $5000 \mathrm{~mJ}$. Other parameters are shown below.

In order to evaluate the performance of our algorithm OACA in terms of energy consumption, we chose two comparison schemes, one is all local computing (ALC: All Local Computing), that is, each device processes all computing tasks in the local computing module. The other is all edge offloading (AEC: All Edge Computing), that is, each device offloads all computing tasks to the edge cloud through the communication module. At the same time, we conduct experiments based on the high rate and low rate of task arrival.
Table 1: Experiment Parameters

\begin{tabular}{cc}
\hline Parameters & Value \\
\hline$\lambda_{i}^{\text {low }}$ & $15-30$ \\
$\lambda_{i}^{\text {high }}$ & $30-45$ \\
$\mu_{i}$ & $180-240$ \\
$\mu_{i}^{l}$ & $230-300$ \\
$\mu$ & 900 \\
$\rho_{i}^{t}$ & $18-30 \mathrm{mw}$ \\
$\rho_{i}^{l}$ & $1^{-7}-2^{-7} \mathrm{~mJ} /$ cycle \\
$\rho_{e}^{c}$ & $2^{-7}-3^{-7} \mathrm{~mJ} /$ cycle \\
\hline
\end{tabular}

\subsection{Experiment Result}

First test the relationship between the number of servers turned on and the total energy consumption of the system. Let the number of running servers vary from 4 to 12 , and observe the total energy consumption of the three algorithms. The results are shown in Figures 5 and 6. Figure 5 is a low rate situation. From Figure 5, we can see that our OACO algorithm has smaller system energy consumption among the three algorithms. In addition, as the number of servers turned on increases, the energy consumption of local computing and edge offloading gradually increases. Our algorithm is a set of optimal solutions for the offloading decision and the number of servers to be turned on based on the minimum energy consumption. The number of servers to be turned on is an optimal certain value (the value in the figure is 5), and the rest of the servers are turned off. So when the number of servers turned on reaches the optimal value, as the number of servers increases, the system energy consumption is basically stable. Figure 6 shows the high-rate case. Obviously, our OACO algorithm among the three algorithms still has a smaller system energy consumption. In addition, compared with Figure 5, the system energy consumption of the three algorithms is higher, and the optimal number of servers opened by the OACO algorithm needs to be more ( 7 in the figure). The reason is because the result in Figure 6 was obtained at a high rate.

Then, test the relationship between the number of IoT devices and the energy consumption of the system. The number of IoT devices varies from 10 to 100 , and the result are shown in Figures 7 and 8 . As the number of IoT devices increases, the total energy consumption of entire system gradually increases. Figure 7 shows the low rate situation. From Figure 7, we can see that among the three algorithms, the AEC algorithm consumes more energy than the ALC algorithm, but our OACO algorithm has a smaller total system energy consumption. Figure 8 is a high-rate situation. From Figure 8 , we can observe that when computing tasks arrive at a high rate, the energy consumption of local computing for all computing tasks is greater than the energy consumption of offloading processing. This is because the faster the task arrives, the more the task volume, and the more energy-consuming local computing and processing. Therefore, the ALC algorithm consumes more energy than the AEC algorithm.

After that, test the impact of the $\alpha$ value on the system energy consumption. Let the value of $\alpha$ change from 0.2 to 0.8 , observe the impact of the three algorithms on the total energy consumption 


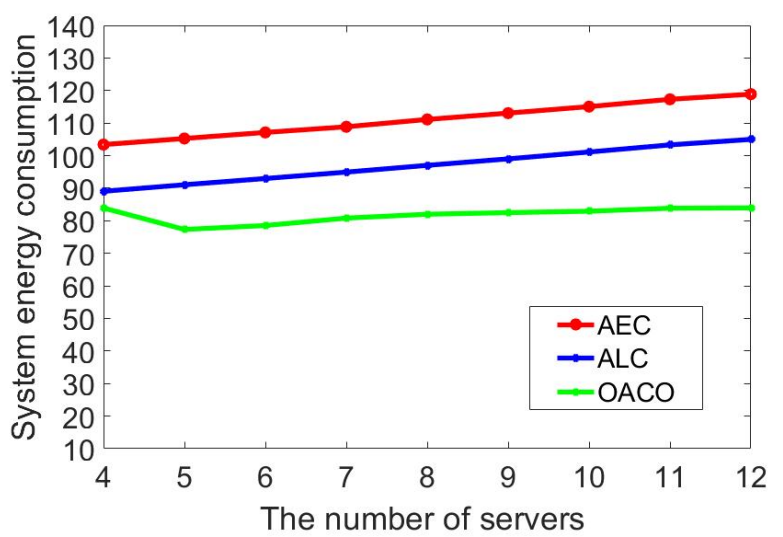

Figure 5: The impact of the number of servers running in the edge cloud on the system energy consumption at low rate.

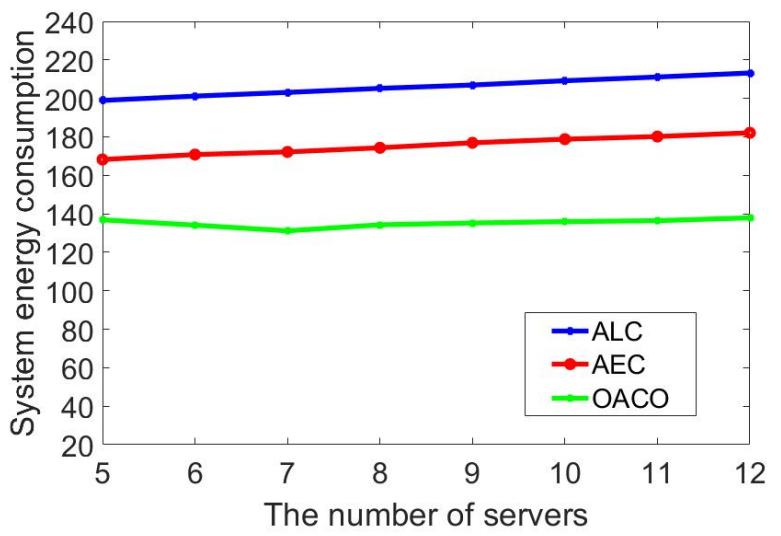

Figure 6: The impact of the number of servers running in the edge cloud on the system energy consumption at low rate.

of entire system, and the results are shown in Figures 9 and 10. $\alpha$ reprsents the weight of the device side. If the value of $\alpha$ is larger, the energy consumption of the device is more important. If the value of $\alpha$ is smaller, the energy consumption of the edge server is more important. Figure 9 shows the low rate situation. When $\alpha$ is at 0.6 , the total energy consumption of the ALC algorithm and the AEC algorithm are the closest. When $\alpha$ is greater than 0.6 , the total system energy consumption of the AEC algorithm is less than that of the ALC algorithm. In our OACO algorithm, as $\alpha$ increases, the total energy consumption of the system also increases. But once it exceeds the critical value (that is, $\alpha$ is 0.6 ), the total energy consumption of the system decreases as $\alpha$ increases. Figure 10 shows the high-rate situation. It can be observed from Figure 10 that when the value of $\alpha$ is greater than 0.5 , the total energy consumption of the system of the AEC algorithm is less than that of the ALC algorithm. And our OACO algorithm still has lower total system energy consumption.

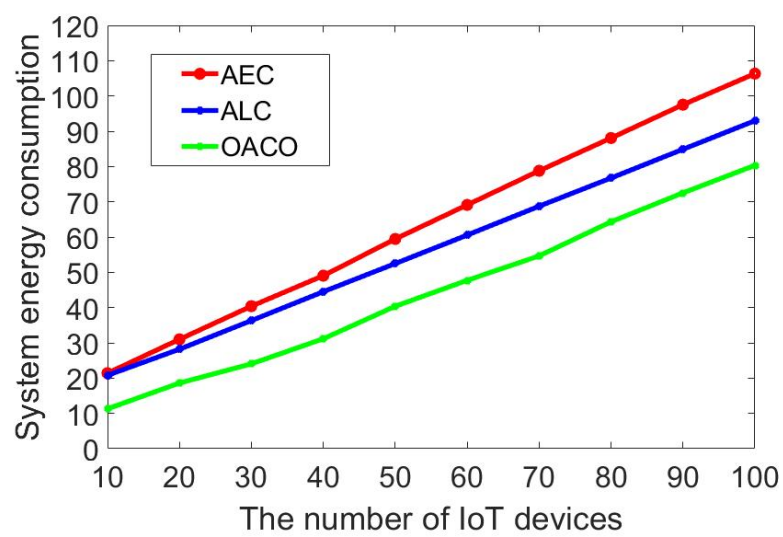

Figure 7: The impact of the number of IoT devices on the system energy consumption at high rate.

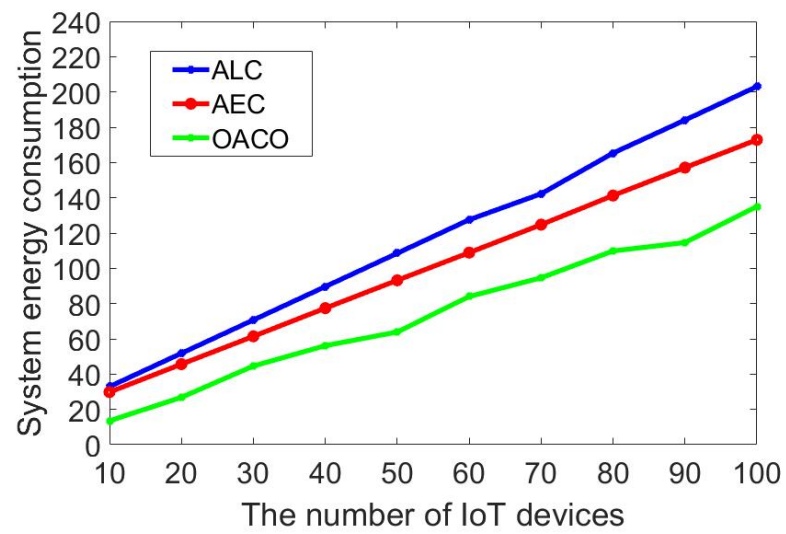

Figure 8: The impact of the number of IoT devices on the system energy consumption at high rate.

\section{CONCLUSION}

This paper proposes a global energy optimization strategy based on delay constraints in an edge computing environment. The service process of the communication module in the IoT device and the service process of the edge cloud are modeled as a queuing network model. This queuing network model is composed of multiple single-server exponential queuing models (M/M/1) and multi-server exponential queuing models $(\mathrm{M} / \mathrm{M} / \mathrm{K})$ to analyze the average delay of the system processing computing tasks. Our strategy also considers the energy consumption of IoT devices and edge cloud, and finds the number of running servers in the edge cloud under the condition of satisfying the delay constraint, so as to achieve the optimal global energy consumption. Experiments show that this strategy is effective for this problem.

In this model, the IoT devices are static. In the future, we can further expand the model to take the mobility of the device into account. Due to the mobility of devices, how many servers will be turned on by the edge cloud and how much computing will be 


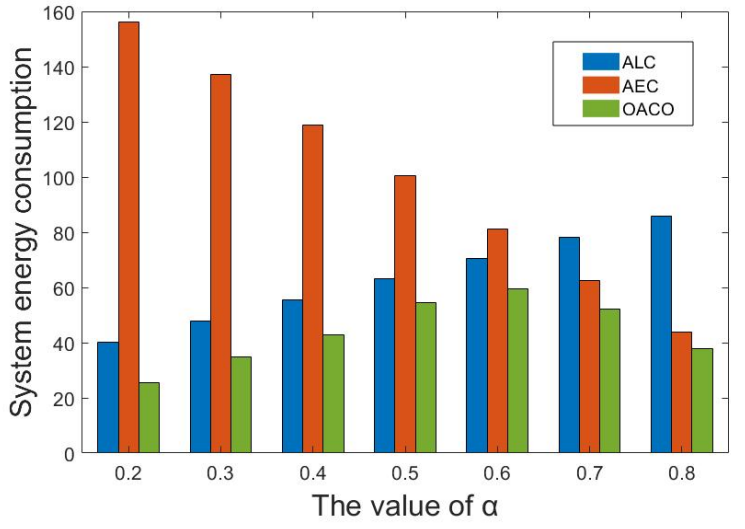

Figure 9: The impact of the value of $\alpha$ on the system energy consumption at low rate.

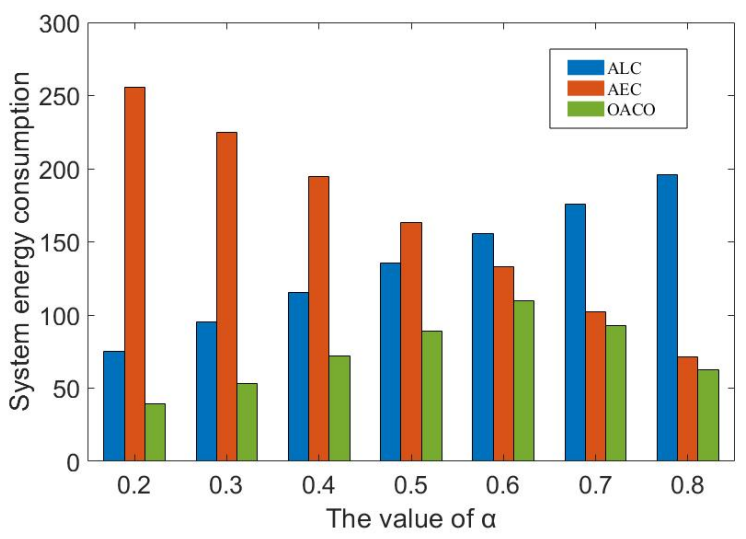

Figure 10: The impact of the value of $\alpha$ on the system energy consumption at high rate.

offloaded by IoT devices will minimize the overall energy consumption of the system.

\section{ACKNOWLEDGMENTS}

This work was supported by the National Natural Science Foundation of China (Grant No. 61672465 and No. 61772472) and Zhejiang Provincial Natural Science Foundation of China (LY15F020027).

\section{REFERENCES}

[1] Suzhi Bi and Ying Jun Zhang. 2018. Computation Rate Maximization for Wireless Powered Mobile-Edge Computing With Binary Computation Offloading. IEEE Transactions on Wireless Communications 17, 6 (2018), 4177-4190. https://doi. org/10.1109/TWC.2018.2821664

[2] Ivona Brandic, Tony Mastelli, and Shijie Liu. 2015. Cloud Computin: Road to energy saving. Global Science 8 (2015), 22-29.

[3] Xu Chen, Lei Jiao, Wenzhong Li, and Xiaoming Fu. 2016. Efficient Multi-User Computation Offloading for Mobile-Edge Cloud Computing. IEEE/ACM Transactions on Networking 24, 5 (2016), 2795-2808. https://doi.org/10.1109/TNET.2015. 2487344

[4] Xing Chen and Guizhong Liu. 2021. Energy-Efficient Task Offloading and Resource Allocation via Deep Reinforcement Learning for Augmented Reality in
Mobile Edge Networks. IEEE Internet of Things fournal 8, 13 (2021), 10843-10856. https://doi.org/10.1109/JIOT.2021.3050804

[5] Laizhong Cui, Chong Xu, Shu Yang, Joshua Zhexue Huang, Jianqiang Li, Xizhao Wang, Zhong Ming, and Nan Lu. 2019. Joint Optimization of Energy Consumption and Latency in Mobile Edge Computing for Internet of Things. IEEE Internet of Things fournal 6, 3 (2019), 4791-4803. https://doi.org/10.1109/JIOT.2018.2869226

[6] Liang Huang, Suzhi Bi, and Ying-Jun Angela Zhang. 2020. Deep Reinforcement Learning for Online Computation Offloading in Wireless Powered Mobile-Edge Computing Networks. IEEE Transactions on Mobile Computing 19, 11 (2020), 2581-2593. https://doi.org/10.1109/TMC.2019.2928811

[7] Nahida Kiran, Chunyu Pan, Sihua Wang, and Changchuan Yin. 2020. Joint resource allocation and computation offloading in mobile edge computing for SDN based wireless networks. Journal of Communications and Networks 22, 1 (2020), 1-11. https://doi.org/10.1109/JCN.2019.000046

[8] Sun Mao, Supeng Leng, Sabita Maharjan, and Yan Zhang. 2020. Energy Efficiency and Delay Tradeoff for Wireless Powered Mobile-Edge Computing Systems With Multi-Access Schemes. IEEE Transactions on Wireless Communications 19, 3 (2020), 1855-1867. https://doi.org/10.1109/TWC.2019.2959300

[9] Yuyi Mao, Changsheng You, Jun Zhang, Kaibin Huang, and Khaled B. Letaief. 2017. A Survey on Mobile Edge Computing: The Communication Perspective. IEEE Communications Surveys Tutorials 19, 4 (2017), 2322-2358. https://doi.org/ 10.1109/COMST.2017.2745201

[10] Minghui Min, Liang Xiao, Ye Chen, Peng Cheng, Di Wu, and Weihua Zhuang. 2019. Learning-Based Computation Offloading for IoT Devices With Energy Harvesting. IEEE Transactions on Vehicular Technology 68, 2 (2019), 1930-1941. https://doi.org/10.1109/TVT.2018.2890685

[11] Zhaolong Ning, Peiran Dong, Xiangjie Kong, and Feng Xia. 2019. A Cooperative Partial Computation Offloading Scheme for Mobile Edge Computing Enabled Internet of Things. IEEE Internet of Things fournal 6, 3 (2019), 4804-4814. https: //doi.org/10.1109/JIOT.2018.2868616

[12] Umber Saleem, Yu Liu, Sobia Jangsher, Xiaoming Tao, and Yong Li. 2020. Latency Minimization for D2D-Enabled Partial Computation Offloading in Mobile Edge Computing. IEEE Transactions on Vehicular Technology 69, 4 (2020), 4472-4486. https://doi.org/10.1109/TVT.2020.2978027

[13] Ali Haider Shamsan and Arman Rasool Faridi. 2018. SDN-Assisted IoT Architecture: A Review. In 2018 4th International Conference on Computing Communication and Automation (ICCCA). 1-7. https://doi.org/10.1109/CCAA.2018.8777339

[14] Mianyang Yao, Long Chen, Tonglai Liu, and Jigang Wu. 2019. Energy Efficient Cooperative Edge Computing with Multi-Source Multi-Relay Devices. In 2019 IEEE 21st International Conference on High Performance Computing and Communications; IEEE 17th International Conference on Smart City; IEEE 5th International Conference on Data Science and Systems (HPCC/SmartCity/DSS). 865-870. https://doi.org/10.1109/HPCC/SmartCity/DSS.2019.00126

[15] Luo Yuchong, Wu Jigang, Wu Yalan, and Chen Long. 2019. Task Scheduling in Mobile Edge Computing with Stochastic Requests and M/M/1 Servers. In 2019 IEEE 21st International Conference on High Performance Computing and Communications; IEEE 17th International Conference on Smart City; IEEE 5th International Conference on Data Science and Systems (HPCC/SmartCity/DSS). 2379-2382. https://doi.org/10.1109/HPCC/SmartCity/DSS.2019.00331

[16] Jiao Zhang, Xiping Hu, Zhaolong Ning, Edith C.-H. Ngai, Li Zhou, Jibo Wei, Jun Cheng, and Bin Hu. 2018. Energy-Latency Tradeoff for Energy-Aware Offloading in Mobile Edge Computing Networks. IEEE Internet of Things fournal 5, 4 (2018), 2633-2645. https://doi.org/10.1109/JIOT.2017.2786343

[17] Tianchu Zhao, Sheng Zhou, Linqi Song, Zhiyuan Jiang, Xueying Guo, and Zhisheng Niu. 2020. Energy-optimal and delay-bounded computation offloading in mobile edge computing with heterogeneous clouds. China Communications 17, 5 (2020), 191-210. https://doi.org/10.23919/JCC.2020.05.015

[18] Anqi Zhu, Songtao Guo, Mingfang Ma, Hao Feng, Bei Liu, Xin Su, Minghong Guo, and Qiucen Jiang. 2019. Computation Offloading for Workflow in Mobile Edge Computing Based on Deep Q-Learning. In 2019 28th Wireless and Optical Communications Conference (WOCC). 1-5. https://doi.org/10.1109/WOCC.2019. 8770689 